\title{
Bilateral subclavian artery stenosis found by inter-arm blood pressure difference during distal pancreatectomy
}

\author{
Do-Hun Kim ${ }^{1}$, Mi-Ja Yun ${ }^{1}$, Hyo-Seok Na ${ }^{2}$, Jung-Won Lee ${ }^{1}$, and Hyo-Ju Hong ${ }^{1}$ \\ Department of Anesthesiology and Pain Medicine, ${ }^{1}$ National Medical Center, Seoul, ${ }^{2}$ Seoul National University Bundang Hospital, \\ Seongnam, Korea
}

Guidelines [1,2] recommend that the blood pressure (BP) should be measured in both arms at the initial assessment of a patient, and that if there is a difference, the arm with the higher pressure should be used for all future measurements. However, $\mathrm{BP}$ is usually measured in the arm that does not have an intravenous (IV) line in it or in the arm that is easier to access during anesthesia in the operating room. In the present case, we report a patient who showed an inter-arm blood pressure difference during an operation. Bilateral filling defects at the os areas of the subclavian arteries and vertebral-subclavian steal were observed on postoperative CT enhanced imaging.

A 74 year-old male (height $152 \mathrm{~cm}$, weight $45.8 \mathrm{~kg}$ ) was scheduled to undergo distal pancreatectomy and left partial adrenalectomy for intraductal papillary mucinous carcinoma of the pancreas. Concentric right ventricular hypertrophy, posterior infarct, and borderline ST elevation were observed on his electrocardiogram (ECG) and the measured left ventricular ejection fraction was $68 \%$ according to an echocardiogram. Preoperative systolic and diastolic $\mathrm{BP}$ and heart rate (HR) measured in the ward were 103-122/55-69 mmHg, and 68-92 beats/min, respectively.

The non-invasive blood pressure (NIBP) was measured in his right upper arm as the IV line was placed in the left arm. General anesthesia was induced with $80 \mathrm{mg}$ of propofol, $40 \mathrm{mg}$ of rocuronium and a target controlled infusion (TCI) of remifentanil (concentration of 3-4 $\mathrm{ng} / \mathrm{ml}$ at the effect site) and then, the patient was maintained on 4-8 vol\% desflurane in oxygen/ air and TCI of remifentanil (concentration of 3-4 $\mathrm{ng} / \mathrm{ml}$ at the effect site). The right radial artery (RRA) and right internal jugular vein were cannulated.

An inverted $\mathrm{T}$ shape incision in the upper abdomen was made and bilateral traction was applied in the slight back up position at 35 min following anesthesia induction. The BP in the RRA decreased to $55 / 39 \mathrm{mmHg}$ at $30 \mathrm{~min}$ after starting the operation in spite of the scanty bleeding from the operating field, with the central venous pressure at $9 \mathrm{cmH}_{2} \mathrm{O}$. The decline of $\mathrm{BP}$ was not reversed by IV ephedrine $10 \mathrm{mg}$, phenylephrine up to $60 \mu \mathrm{g}$ and dopamine infusion $10 \mu \mathrm{g} / \mathrm{kg} / \mathrm{min}$. Intermittent IV bolus doses of phenylephrine (up to $40 \mu \mathrm{g}$ ) and epinephrine (up to $20 \mu \mathrm{g}$ ) were tried: these measures increased HR from 67-95/min to $92-$ $123 / \mathrm{min}$, but did not restore the reduced BP in the RRA (5895/42-64 mmHg). The patient was placed in the Trendelenburg position and $0.04 \mu \mathrm{g} / \mathrm{kg} / \mathrm{min}$ of norepinephrine was infused for $20 \mathrm{~min}$, but it did not increase the arterial BP in the RRA. Radial artery cannulation and NIBP were performed on the left side of the arm and the measured BPs were higher than those of the right arm (NIBP, 64/48 vs. $108 / 50 \mathrm{mmHg}$ in the right arm vs. left arm; arterial BP, 59/41 vs. $121 / 49 \mathrm{mmHg}$ on the RRA vs. LRA). Arterial BP in the LRA was monitored on the left arm after that. The infusion rates of dopamine and norepinephrine were tapered off. The arterial BP in the LRA was maintained as $98-129 / 48-57 \mathrm{mmHg}$ throughout the operation and central venous pressure ranged from 8 to $11 \mathrm{cmH}_{2} \mathrm{O}$ throughout the operation. During the $3 \mathrm{hr} 15 \mathrm{~min}$ of anesthesia time, $1900 \mathrm{ml}$

Corresponding author: Mi-Ja Yun, Ph.D., Department of Anesthesiology and Pain Medicaine, National Medical Centar, 243, Eulji-ro, Jung-gu, Seoul 100-799, Korea. Tel: 82-2-2260-7374, Fax: 82-2-2262-4766, E-mail: anemjy@gmail.com

(c) This is an open-access article distributed under the terms of the Creative Commons Attribution Non-Commercial License (http:// creativecommons.org/licenses/by-nc/3.0/), which permits unrestricted non-commercial use, distribution, and reproduction in any medium, provided the original work is properly cited. 

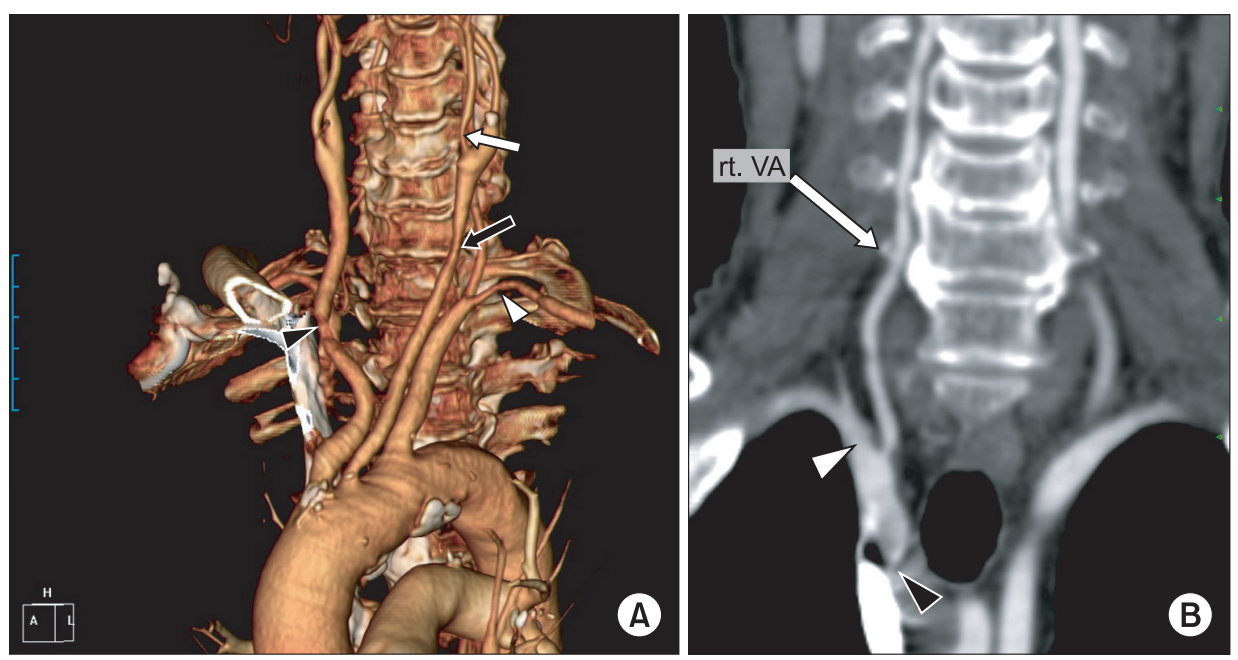

Fig. 1. (A) Three-dimensional computed tomography angiogram of the thoracic aorta and branches shows the occlusion of the os area of the right subclavian artery (black arrow head), stenosis of the left subclavian artery (white arrow head), left common carotid artery (black arrow), and left internal carotid artery (white arrow). (B) Computed tomography enhanced coronal multiplana reformatting image (MPR) of thoracic aorta and branches. The os area of the right subclavian artery is occluded (black arrow head), so the right subclavian artery (white arrow head) is visualized by the reverse arterial flow from the vertebral artery (white arrow).

of crystalloid and $550 \mathrm{ml}$ of colloid were administered. The estimated blood loss was $200 \mathrm{ml}$ and the urine output was $750 \mathrm{ml}$. The patient recovered smoothly from the general anesthesia and was discharged 13 days after the operation without complications. Three months after the operation, we observed a focal filling defect in the os area of the right subclavian artery (suggestive of arterial occlusion by a thrombus), and multifocal atheromatic stenosis at the ascending aorta, os area of the left subclavian artery, left common carotid artery, and internal carotid artery on his thoracic 3-dimensional computed tomography (3D CT) angiography (Fig. 1A). We also recognized vertebral subclavian steal on the CT-enhanced image (Fig. 1B).

In the present case, bilateral BP measurements were not performed either at the ward or at the operating room before anesthesia. The absence of patient's symptoms from the subclavian arterial stenosis and the delay in the physicians' suspicions and detection of the inter-arm BP difference caused the unnecessary administration of inotropic drugs during anesthesia. In the present case, delayed retrograde down-flow from the right vertebral artery to the right subclavian artery was noted and it might have contributed to the absence of central nervous system symptoms such as dizziness, motor and sensory deficits, vertigo, and dysarthria.

The predominant cause of inter-arm BP differences is atherosclerotic obstruction and it is mainly due to subclavian artery stenosis [3]. Shadman et al. [3] reported that significant subclavian stenosis is present in $7 \%$ of the clinical population and that subclavian artery stenosis (inter-arm systolic BP differ- ence $\geq 15 \mathrm{mmHg}$ ) is correlated with current and past smoking histories, systolic BP, HDL levels (inversely), and the presence of peripheral arterial disease. And they proposed that bilateral BP measurements should routinely be performed in patients with an elevated risk profile, both to screen for subclavian artery stenosis, and to avoid missing hypertension or peripheral arterial disease. In this case, the actions of the drug were insignificant in comparison to its actions on average healthy adults although the $\mathrm{BP}$ on the left side was higher than that of the right. This possibly suggests the presence of bilateral stenosis of the subclavian artery with more stenosis on the right side.

Asymmetrical atheromatous narrowing of the subclavian or brachial arteries would be expected to be more common with increasing age, leading to lower pressures in the arteries distal to the narrowing, and significant inter-arm BP differences $[3,4]$. In the present case, the patient' age and ECG findings could have been risk factors for the existence of other vascular disease and the inter-arm BP difference.

Inappropriate management of pseudohypotension or the masked hypertension based on erroneous clinical decisions can increase the risk of cerebrovascular, cardiac, or renal complications in the future [5]. For the patient who is scheduled to undergo coronary artery bypass graft, measurement of the bilateral brachial $\mathrm{BP}$ and intra-operative doppler flow in the left internal mammary artery graft are essential to exclude coronary subclavian steal syndrome, especially in patients with diffuse cerebrovascular and peripheral vascular disease. 


\section{References}

1. European Society of Hypertension-European Society of Cardiology Guidelines Committee. 2003 European Society of HypertensionEuropean Society of Cardiology guidelines for the management of arterial hypertension. J Hypertens 2003; 21: 1011-53.

2. National Institute for Health and Clinical Excellence. Hypertension: the clinical management of primary hypertension in adults, CG127. London: National Institute for Health and Clinical Excellence. 2011.

3. Shadman R, Criqui MH, Bundens WP, Fronek A, Denenberg JO, Gamst AC, et al. Subclavian artery stenosis: prevalence, risk factors, and association with cardiovascular diseases. J Am Coll Cardiol 2004; 44: 618-23.

4. Lane D, Beevers M, Barnes N, Bourne J, John A, Malins S, et al. Inter-arm differences in blood pressure: when are they clinically significant? J Hypertens 2002; 20: 1089-95.

5. Frank SM, Norris EJ, Christopherson R, Beattie C. Right- and left-arm blood pressure discrepancies in vascular surgery patients. Anesthesiology 1991; 75: 457-63. 